\title{
Mechanism Design and Dynamical Modelling of a Flexible-Wire-Walking Robot with Swinging-Arms and Rotational Pole
}

\author{
Yonghua Huang*, Yaxiong Chen, Changsheng Wang and Hao Huang \\ School of Mechanical and Electrical Engineering, Guilin University of Electronic Technology, Guilin, China, 541004 \\ ${ }^{*}$ Corresponding author
}

\begin{abstract}
In order to investigate the principles of a wire walker who maintains balance by rotating or moving a pole left and right, a novel single wheel robot that can runs on a flexible wire was designed and its dynamics was studied. The robot features a couple of parallel swinging arms and a rotational pole. Under the precondition of pure rolling of the single wheel that runs on a flexible wire, a dynamical model for the system was developed by use of Lagrange equation. The results illustrated that the robot was an under-actuated system consisting of seven DOF (degree of freedom) and there was three driving-torque inputs in the system. By using the model, an inverse dynamics simulation of balanced moving was performed, and through the comparisons of the amount of driving work and system's energy, the results of the model analysis was testified.
\end{abstract}

Keywords-wire-walking robot; flexible wire; dynamical model; lagrange equation

\section{INTRODUCTION}

Wire-walking robots are commonly denoted the kind of mobile mechanical systems that can imitate a human walker running above a tight wire by rolling a pole up and down or moving a translational mass left and right to keep balance. In recent years, mechanism designing and system modeling are two attractive topics in wire- walking robot research. For example, in [1], Zhou etc. proposed a two wheels wirewalking robot inspiring by the principle of gyroscopic stability. And in [2]-[6], Lu etc. presented a wire-walking robot with a rotating pole. Moreover, in [3], Lu developed a dynamical model by use of Lagrange equations and discussed the balance controller for the system. In [7]-[8], Guo etc. suggested a wire-walking robot that featured a rotational pole and a translational pole. As an extension, dynamical model based on Lagrange equations and balanced controllers for the robot system are also under the consideration in [8]. Accounting for the manners of that a human wire-walker, the wire-walking robot proposed in [7][8] can approximately imitated the balance regulating activities of a real human walker, but its translational pole is driven by a parallelogram mechanism that is rotating about a horizontal axis, thus there may be some difference to a vertically swing arms of a real wire-walker. Moreover, the rotational pole and the translational pole in [7]-[8] are structural independent for each other, which should be a lot of difference to a real human wire-walker. In addition, in the presented researches, most of the work concerning on modelling a wire-walking robot by neglecting the flexibility of the support wire, so their model's accuracy would be insufficient when the system's dynamical analysis is performed.

For investigating the principle of a real human wirewalker more effectively, a novel wire-walker with two swing-arms and a rotational pole is proposed in this paper. The skeletons of the paper is as following: at the beginning, the mechanism of the proposed wire-walking robot is demonstrated in detail; and next, a dynamical model for the system is developed; at the end, considering the real situation that the robot walker was running on a flexible wire, a numerical simulation about the inverse dynamics was then performed to testify the effectiveness of the proposed model.

\section{GENERAL SCHEME}

In [7] [8], a parallelogram mechanism was exploited in the wire-walking robot to achieve the function of the two arms of a real human wire-walker. However, as mentioned above, due to the parallelogram mechanism rotating about a horizontal axis, it is a little difference to a vertically swing arms of a human wire-walker, hence in this paper, an kind of innovated parallel swinging arms that can rotate about a vertical axis was then seriously under consideration.

\section{A. Mechanical Structure}

The considering wire-walking robot consists of five parts, that is, a supporting frame, a running wheel, two swinging arms, a link pole and a rotational pole. Figure. $1 \sim$ Figure. 2 is the detail mechanism of the system. 


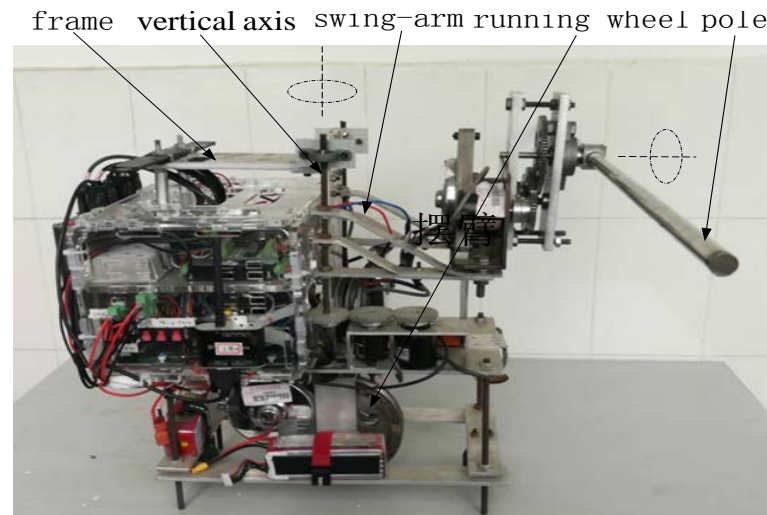

FIGURE I. PRINCIPLE PROTOTYPE OF THE WIRE-WALKING ROBOT

As seen in Figure 1 Figure 2, a single running wheel was set at the bottom of the robot to imitate the action of a human walker in single leg supporting phase. There a $\mathrm{V}$ groove was engraved on the circular outline of the wheel, therefore the supporting wire can be insert into groove to avoid the robot slipping out of the wire. Two swinging arms horizontally mounted on the robot's frame, which served as a wirewalker's vertically swing arms. A rotational pole was set at the end of the two arms, which served as the balanced long pole of the real wire- walker. In addition, the swinging arms, the rotational pole and the running wheel are all driven by their individual motor.

\section{B. Working Principle}

The working principle of the proposed wire-walking robot can be summarized as follow:

When the COM (center of mass) of the robot drifts right or left, the robot would drive the rotational pole to rotate in the direction of drifting of the COM to shift it. Simultaneously, the swing arms are also driven to rotate vertically to the opposite side of the drifting of the COM, and finally as a reaction, the COM of the robot then returns to the equilibrium position.

Similarly, when the COM of the robot drifts forward or backward, the robot would accelerate or decelerate its wheel in the direction of the drifting of the COM to shift it. Through this regulation, the COM of the robot would turn back balance.

\section{DYNAMICAL MODEL}

In this section, a dynamical model for the robot system is developed by using Lagrange equation.

\section{A. Coordination Settings}

Let the supporting frame be denoted as $B_{1}$, the single wheel as $B_{2}$, the swinging arms as $B_{3}$, the link pole as $B_{4}$ and the rotational pole as $B_{5}$. The coordinates settings of the robot are showed in Figure. 2.
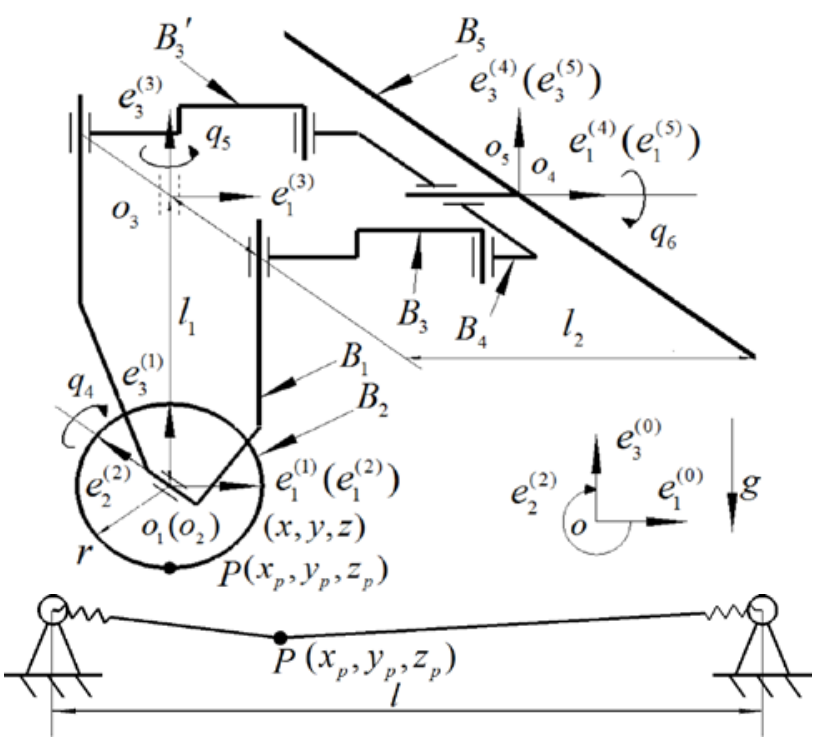

FIGURE II. SCHEMATIC DIAGRAM OF THE WIRE-WALKING ROBOT

$O-\boldsymbol{e}_{1} \boldsymbol{e}_{2} \boldsymbol{e}_{3}\{0\}$ is the global coordinate system fixed on the ground;

$O_{1}-\boldsymbol{e}_{1}^{(1)} \boldsymbol{e}_{2}^{(1)} \boldsymbol{e}_{3}^{(1)}\{1\}$ is the coordinate system of $B_{1}$ and its origin is the geometric center of $B_{2}$;

$O_{2}-\boldsymbol{e}_{1}^{(2)} \boldsymbol{e}_{2}^{(2)} \boldsymbol{e}_{3}^{(2)}\{2\}$ is the coordinate system of $B_{2}$ and its origin is the same as $\{1\}$

$O_{3}-\boldsymbol{e}_{1}^{(3)} \boldsymbol{e}_{2}^{(3)} \boldsymbol{e}_{3}^{(3)}\{3\}$ is the coordinate system of $B_{3}$ and the origin locates at center of the two arms.

$O_{4}-\boldsymbol{e}_{1}^{(4)} \boldsymbol{e}_{2}^{(4)} \boldsymbol{e}_{3}^{(4)}\{4\}$ is the coordinate system of $B_{4}$ and the origin locates at its geometric center.

$O_{5}-\boldsymbol{e}_{1}^{(5)} \boldsymbol{e}_{2}^{(5)} \boldsymbol{e}_{3}^{(5)}\{5\}$ is the coordinate system of $B_{4}$ and the origin locates at its geometric center.

\section{B. Kinetic Model}

Supposing the situation that the wire-walking robot ran on a flexible wire without any slipping and there was no twist on the wire, then the angular velocity of $B_{1}$ can be represented as:

$$
\boldsymbol{\omega}_{B 1}^{(1)}=c_{3} \dot{q}_{2} \boldsymbol{e}_{1}^{(1)}+\dot{q}_{3} \boldsymbol{e}_{2}^{(1)}+S_{3} \dot{q}_{2} \boldsymbol{e}_{3}^{(1)},
$$

where $\boldsymbol{e}_{i}^{(j)}(i=1,2,3, j=1,2, \cdots)$ is the ith base vector of $\{j\} . \dot{q}_{i}(i=1,2,3)$ is the ith Eular angular rate of $B_{1}$; $s_{i}=\sin \left(q_{i}\right), c_{i}=\cos \left(q_{i}\right)(i=1,2, \cdots)$.

Because $B_{2}$ rotates about $B_{1}$, angular velocity of $B_{2}$ can be calculated as:

$$
\boldsymbol{\omega}_{B 2}^{(1)}=c_{3} \dot{q}_{2} \boldsymbol{e}_{1}^{(1)}+\left(\dot{q}_{3}+\dot{q}_{4}\right) \boldsymbol{e}_{2}^{(1)}+S_{3} \dot{q}_{2} \boldsymbol{e}_{3}^{(1)},
$$


where $\dot{q}_{4}$ denotes the angular rate of $B_{2}$.

$$
\boldsymbol{v}_{C 3}^{(1)}={ }^{1} \boldsymbol{R}_{0} \cdot \boldsymbol{v}_{C 2}^{(0)}+\boldsymbol{\omega}_{B 1}^{(1)} \times \boldsymbol{l}_{o 3}^{(1)}+{ }^{1} \boldsymbol{R}_{3} \cdot\left(\boldsymbol{\omega}_{B 3}^{(3)} \times \boldsymbol{l}_{C 3}^{(3)}\right),
$$

Similarly, $B_{3}$ rotates about $B_{1}$, so the angular velocity of $B_{3}$ can be calculated as:

$\boldsymbol{\omega}_{B 3}^{(3)}=\left(c_{3} c_{5} \dot{q}_{2}+s_{5} \dot{q}_{3}\right) \boldsymbol{e}_{1}^{(3)}+\left(c_{5} \dot{q}_{3}-c_{3} s_{5} \dot{q}_{2}\right) \boldsymbol{e}_{2}^{(3)}+\left(s_{3} \dot{q}_{2}+\dot{q}_{5}\right) \boldsymbol{e}_{3}^{(3)}$

where $\dot{q}_{5}$ is the angular rate of $B_{3}$.

The two swing arms and the link pole form a parallelogram mechanism, so its angular velocity satisfies: $\boldsymbol{\omega}_{B 4}^{(4)}=\boldsymbol{\omega}_{B 1}^{(1)}$.

Moreover, $B_{5}$ about $B_{4}$, so its angular velocity can be:

$$
\boldsymbol{\omega}_{B 5}^{(5)}=\left(c_{3} \dot{q}_{2}+\dot{q}_{6}\right) \boldsymbol{e}_{1}^{(1)}+\dot{q}_{3} \boldsymbol{e}_{2}^{(1)}+s_{3} \dot{q}_{2} \boldsymbol{e}_{3}^{(1)} .
$$

According to the presumption, the robot ran on a flexible wire without slipping, so the coordinations $\left(\begin{array}{lll}x_{p} & y_{p} & z_{p}\end{array}\right)^{T}$ of the point $P$ and the coordination $\left(\begin{array}{lll}x & y & z\end{array}\right)^{T}$ of the geometric center of $B_{2}$ should satisfies the following constrains:

$$
\begin{gathered}
x=x_{p}=\sqrt{\left(q_{3}+q_{4}\right)^{2} r^{2}-\left(y_{p}^{2}+z_{p}^{2}\right)}, \\
y=y_{p}-r s_{2}, \quad z=z_{p}+r c_{2},
\end{gathered}
$$

where $r$ denotes the radius of the running wheel.

Supposing the first time derivatives of (5) (7) were taken, then the following expression would be get

$$
\begin{gathered}
\dot{x}=\dot{x}_{p}=\left(r^{2}\left(q_{3}+q_{4}\right)\left(\dot{q}_{3}+\dot{q}_{4}\right)-y_{p} \dot{y}_{p}-z_{p} \dot{z}_{p}\right) / x, \\
\dot{y}=\dot{y}_{p}-r c_{2} \dot{q}_{2}, \dot{z}=\dot{z}_{p}-r s_{2} \dot{q}_{2} .
\end{gathered}
$$

Let the velocity of the COM of $B_{4}$ be

$$
\boldsymbol{v}_{C 2}^{(0)}=\dot{x} \boldsymbol{e}_{1}^{(0)}+\dot{y} \boldsymbol{e}_{2}^{(0)}+\dot{z} \boldsymbol{e}_{3}^{(0)} .
$$

Considering the principle of the relative motion, the velocities of the COM of $B_{1}, B_{3}, B_{4}$ and $B_{5}$ can be calculated as:

$$
\boldsymbol{v}_{C 1}^{(1)}={ }^{1} \boldsymbol{R}_{0} \cdot \boldsymbol{v}_{C 2}^{(0)}+\boldsymbol{\omega}_{B 1}^{(1)} \times \boldsymbol{l}_{C 1}^{(1)},
$$

$$
\boldsymbol{v}_{C k}^{(1)}={ }^{1} \boldsymbol{R}_{0} \cdot \boldsymbol{v}_{C 2}^{(0)}+\boldsymbol{\omega}_{B 1}^{(1)} \times \boldsymbol{l}_{o 3}^{(1)}+{ }^{1} \boldsymbol{R}_{3} \cdot\left(\boldsymbol{\omega}_{B 3}^{(3)} \times \boldsymbol{l}_{o 4}^{(3)}\right)+{ }^{1} \boldsymbol{R}_{4} \cdot\left(\boldsymbol{\omega}_{B 4}^{(4)} \times \boldsymbol{l}_{C k}^{(4)}\right)
$$

$$
(k=4,5)
$$

where ${ }^{j} \boldsymbol{R}_{i}(i, j=1,2, \cdots)$ denotes the rotation transform matric from $\{i\}$ to $\{j\}, \boldsymbol{I}_{C i}^{(i)}(i=1,3,4,5)$ is the position vector in $\{i\}$ from $o_{i}$ to the COM of $B_{i} ; \boldsymbol{l}_{o i}^{(1)}(i=3,4,5)$ is the position vector in $\{1\}$ from the geometric center of $B_{2}$ to the geometric center of $B_{i}(i=3,4,5)$.

\section{Kinetic Energy and Potential Energy}

Considering the former derived $\boldsymbol{\omega}_{B i}^{(j)}(i, j=1, \cdots, 5)$ and $\boldsymbol{v}_{C i}^{(k)}(i=1, \cdots, 5 ; k=0,1)$, then the kinetic energy of the system can be calculated as:

$$
T=\sum_{i=1}^{5}\left(\left(\boldsymbol{\omega}_{B i}^{(j)}\right)^{T} \boldsymbol{J}_{B i}\left(\boldsymbol{\omega}_{B i}^{(j)}\right)+\left(\boldsymbol{v}_{C i}^{(k)}\right)^{T} \boldsymbol{M}_{B i}\left(\boldsymbol{v}_{C i}^{(k)}\right)\right) / 2,
$$

where $\boldsymbol{J}_{B i}\left(\boldsymbol{M}_{i}\right)(i, j=1,2, \cdots, 5)$ represents the inertial matric (mass matrix) of $B_{i}(i=1,2, \cdots, 5)$, respectively.

Let the wire's length be denoted as $l$, then the elongation of the flexible wire can be represented as:

$$
\Delta l=\left(r\left(q_{3}+q_{4}\right)+\sqrt{\left(l-x_{p}\right)^{2}+\left(y_{p}^{2}+z_{p}^{2}\right)}\right)-l,
$$

Considering the height of the integrated COM of $B_{1}$ and $B_{i}(i=3,4,5)$ in $\{1\}$, it is clear that:

$$
\boldsymbol{h}_{C}^{(1)}=\left(m_{1} \boldsymbol{I}_{C 1}^{(1)}+\sum_{i=3}^{5} m_{i}\left(\boldsymbol{I}_{o i}^{(1)}+{ }^{1} \boldsymbol{R}_{i} \boldsymbol{l}_{C i}^{(i)}\right)\right) / \sum_{i=1}^{5} m_{i},
$$

$$
\boldsymbol{h}_{C}^{(0)}=\boldsymbol{I}_{P}^{(0)}+{ }^{0} \boldsymbol{R}_{2} \cdot \boldsymbol{l}_{P}^{(2)}+{ }^{0} \boldsymbol{R}_{1} \cdot \boldsymbol{h}_{C}^{(1)},
$$

where $\boldsymbol{h}_{C}^{(i)}$ ( $i=0,1$ ) denotes the height of the integrated COM in $\{1\}, \boldsymbol{I}_{P}^{(j)}(j=0,2)$ is the position vector of point $P$ in $\{j\}$.

With (17)and(19), the potential energy of the system can be calculated as: 


$$
\boldsymbol{U}=\left(k \Delta l^{2}+\left(g \sum_{i=1}^{5} m_{i}\right)\left(\left(\boldsymbol{e}_{1}^{(0)}\right)^{T} \boldsymbol{h}_{C}^{(0)}\right)^{2}\right) / 2
$$

where $k$ denotes the stiffness coefficient of the wire, $g$ denotes the acceleration constant of the gravity.

\section{Dynamical Model}

Considering the form of Lagrange equation:

$$
\frac{d}{d t}\left(\frac{\partial T}{\partial \dot{q}_{\sigma}}\right)-\frac{\partial T}{\partial q_{\sigma}}+\frac{\partial U}{\partial q_{\sigma}}=\tau_{\sigma}(\sigma=1,2, \cdots)
$$

where $q_{\sigma}$ are the generalized coordinates of the system; $\tau_{\sigma}$ is the $\sigma$ th driving force, and then substituting (16) and (20) into (21), finally the system's dynamics can be get as:

$$
D(\boldsymbol{q}) \ddot{q}+C(\boldsymbol{q}, \dot{q}) \dot{q}+G(\boldsymbol{q})=\boldsymbol{\tau},
$$

where $\boldsymbol{D}(\boldsymbol{q}) \in \mathfrak{R}^{7 \times 7}, \boldsymbol{C}\left(\boldsymbol{q}, \dot{\boldsymbol{q}}_{\theta}\right) \in \mathfrak{R}^{7 \times 7}$, and $\boldsymbol{G}(\boldsymbol{q}) \in \mathfrak{R}^{7 \times 1}$ denotes the inertia, the centripetal-Coriolis, and the gravity terms; $\boldsymbol{q} \in \mathfrak{R}^{7 \times 1}$ are the generalized coordinates, and

$$
\boldsymbol{q}=\left(\begin{array}{lllllll}
y_{p} & z_{p} & q_{2} & q_{3} & q_{4} & q_{5} & q_{6}
\end{array}\right)^{T},
$$

and

$$
\boldsymbol{\tau}=\left(\begin{array}{lllllll}
0 & 0 & 0 & 0 & \tau_{4} & \tau_{5} & \tau_{6}
\end{array}\right)^{T} .
$$

Equation (22) shows the wire-walking robot is an underactuated system with seven independent velocities, and here the displacements $\left(y_{P}, z_{P}\right)$ of the geometric center of point $P$, and the roll angle $\left(q_{2}\right)$ and the pitch angle $\left(q_{3}\right)$ of $B_{1}$ are all under-actuated. Equation (22) also shows that there are three driving torque inputs on $q_{i}(i=4,5,6)$, so the robot can regulate the control-force $\tau_{i}(i=4,5,6)$ to achieve the balanced motion.

\section{INVERSE DyNAMics SimUlations}

Subsequently, inverse dynamics simulations of the

\begin{tabular}{|c|c|c|}
\hline Physical meanings & Sym & Value \\
\hline Radius of $B_{1}(\mathrm{~m})$ & $r$ & 0.10 \\
\hline $\begin{array}{l}\text { Length of the } \\
\text { wire }(\mathrm{m})\end{array}$ & l & 3.00 \\
\hline $\begin{array}{c}\text { Distance from } \\
o_{1}\left(o_{3}\right) \text { to } o_{3}\left(o_{4}\right)(\mathrm{m})\end{array}$ & $l_{1}\left(l_{2}\right)$ & $0.42(0.28)$ \\
\hline $\begin{array}{c}\text { COM of } \\
B_{1}\left(B_{3}, B_{4}\right)(\mathrm{m})\end{array}$ & $\begin{array}{c}l_{C 1}^{(1)} \\
\left(l_{C 3}^{(3)}, l_{C 4}^{(4)}\right)\end{array}$ & 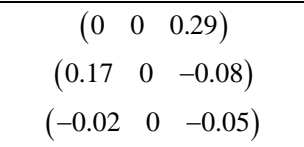 \\
\hline $\begin{array}{c}\text { Mass of } B_{i} \\
(i=1 \sim 5)(\mathrm{kg})\end{array}$ & $\begin{array}{c}m_{i} \\
(i=1 \sim 5)\end{array}$ & $\begin{array}{c}6.57 \\
(1.00,0.41,0.15,3.20)\end{array}$ \\
\hline $\begin{array}{c}\text { Inertia matrix of } \\
B_{i}(i=1 \sim 5)\left(\mathrm{kg} \cdot \mathrm{m}^{2}\right)\end{array}$ & $\begin{array}{c}J_{B i} \\
(i=1 \sim 5)\end{array}$ & $\begin{array}{rll}\operatorname{diag}(0.02 & 0.03 & 0.15) \\
(\operatorname{diag}(0.05 & 0.02 & 0.05), \\
\operatorname{diag}(0.01 & 0.02 & 0.02), \\
\operatorname{diag}(0.02 & 0.01 & 0.02), \\
\operatorname{diag}(0.80 & 0.02 & 0.80))\end{array}$ \\
\hline Gravity constant $\left(\mathrm{m} / \mathrm{s}^{2}\right)$ & $g$ & 9.8 \\
\hline $\begin{array}{l}\text { Wire's stiffness } \\
\text { coefficient }(\mathrm{N} / \mathrm{m})\end{array}$ & $k$ & 5000 \\
\hline
\end{tabular}
balanced motion for the robot was performed.

Table I shows the physical parameters of the robot system which would be used in the simulations.
TABLE I. PHYSICAL PARAMETERS

The simulation is perform with three steps.

\section{- Step 1: trajectory planning}

The trajectory of the roll angle and pitch angle of $B_{1}$ was set as following:

$$
q_{i}(t)=A_{i} e^{-\sigma_{i} t} \sin \left(\omega_{i} t+\varphi_{i}\right)(i=2,3) ; q_{5}(t)=q_{2}(t) ;
$$

where $A_{2}=2 \pi / 180, \sigma_{2}=\sigma_{3}=-0.8, \omega_{2}=2 \pi, \varphi_{2}=\pi / 2$, $A_{3}=5 \pi / 180, \omega_{3}=0.4 \pi, \varphi_{3}=\pi / 6$.

\section{Step 2: motion state calculating}

Considering the first four items of the dynamics that is shown in (22):

$$
\boldsymbol{D}_{u 1}(\boldsymbol{q}) \ddot{\boldsymbol{q}}_{u}+\boldsymbol{D}_{s 1}(\boldsymbol{q}) \ddot{\boldsymbol{q}}_{s}+\boldsymbol{F}_{1}(\boldsymbol{q}, \dot{\boldsymbol{q}})=\mathbf{0}
$$

where $\boldsymbol{F}_{1}(\boldsymbol{q}, \dot{\boldsymbol{q}}) \in \mathfrak{R}^{4 \times 1}$ denotes the first four items of the vector $(\boldsymbol{C}(\boldsymbol{q}, \dot{\boldsymbol{q}}) \dot{\boldsymbol{q}}+\boldsymbol{G}(\boldsymbol{q})) ; \boldsymbol{D}_{u 1}(\boldsymbol{q})\left(\boldsymbol{D}_{s 1}(\boldsymbol{q})\right)$ is a sub-matrix of $\boldsymbol{D}(\boldsymbol{q})$, $\ddot{\boldsymbol{q}}_{u}=\left(\begin{array}{llll}\ddot{x}_{P} & \ddot{y}_{P} & \ddot{q}_{4} & \ddot{q}_{6}\end{array}\right)^{T}, \ddot{\boldsymbol{q}}_{s}=\left(\begin{array}{lll}\ddot{q}_{2} & \ddot{q}_{3} & \ddot{q}_{5}\end{array}\right)^{T}$, it is clear that

$$
\ddot{\boldsymbol{q}}_{s}=-\left(\boldsymbol{D}_{u}(\boldsymbol{q})\right)^{-1} \boldsymbol{F}_{1}(\boldsymbol{q}, \dot{\boldsymbol{q}}) .
$$

According to (24), the angular rate $\dot{\boldsymbol{q}}_{s}$ and the angle $\boldsymbol{q}_{s}$ can be get by the numerical time integration.

\section{Step 3: driving torque calculating}

Considering the later three items of the dynamics in (23), it is obviously that: 


$$
\boldsymbol{\tau}_{a}=\boldsymbol{D}_{u 2}(\boldsymbol{q}) \ddot{\boldsymbol{q}}_{u}+\boldsymbol{D}_{\mathrm{s} 2}(\boldsymbol{q}) \ddot{\boldsymbol{q}}_{s}+\boldsymbol{F}_{2}(\boldsymbol{q}, \dot{\boldsymbol{q}}),
$$

where $\boldsymbol{F}_{2}(\boldsymbol{q}, \dot{\boldsymbol{q}}) \in \mathfrak{R}^{3 \times 1}$ denotes the remaining three items of the vector $(\boldsymbol{C}(\boldsymbol{q}, \dot{\boldsymbol{q}}) \dot{\boldsymbol{q}}+\boldsymbol{G}(\boldsymbol{q}))$, and $\boldsymbol{D}_{u 2}(\boldsymbol{q})\left(\boldsymbol{D}_{\mathrm{s} 2}(\boldsymbol{q})\right)$ is a submatrix of $\boldsymbol{D}(\boldsymbol{q}), \boldsymbol{\tau}_{a}=\left(\begin{array}{lll}\tau_{4} & \tau_{5} & \tau_{6}\end{array}\right)^{T}$.

Figure 3 shows the desired trajectory of $\boldsymbol{q}_{\mathrm{s}}$ in the inverse dynamics simulation.

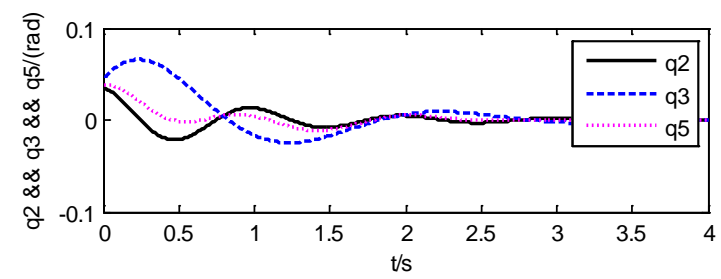

FIGURE III. THE PLANNING TRAJECTORY

Figure 4 demonstrates the acceleration of $B_{1}$, the angular acceleration of $B_{2}$ and the angular acceleration of $B_{5}$.

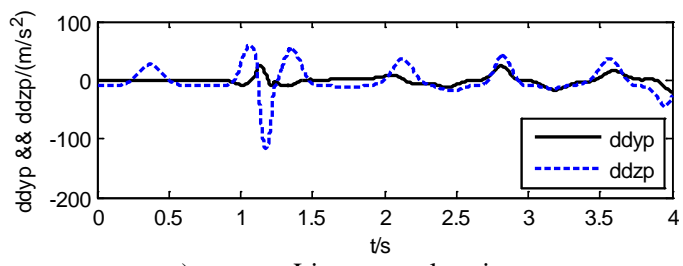

a) Linear acceleration

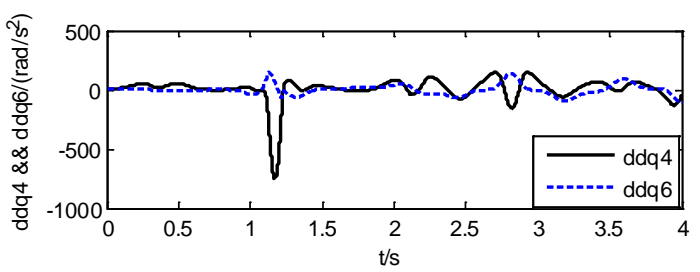

b) Angular acceleration

FIGURE IV. THE ACCELERATION

Figure 5 demonstrates the linear velocities of the point $P$, and the angular rate of $B_{2}$ and $B_{5}$. It was worth noting that the curves that were showed in Figure 5 were obtained through the numerical integration of the data in Figure. 4.

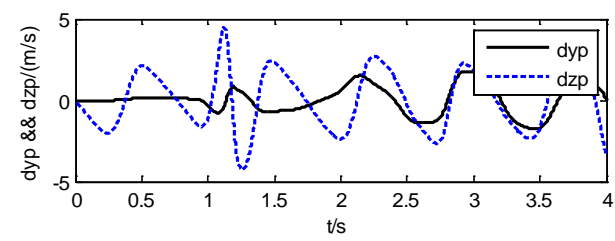

a)

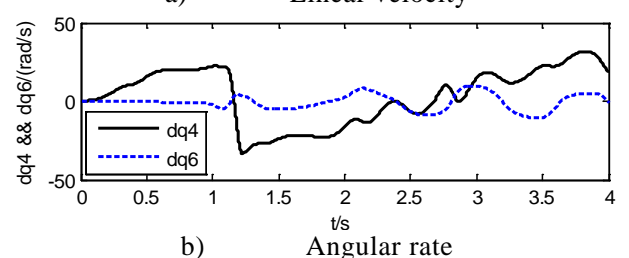

b) Angular rate

FIGURE V. THE LINEAR VELOCITY AND ANGULAR RATE
Figure 6 demonstrates the coordination of the point $P$ and the rotational angle of $B_{2}$ and $B_{5}$. Similarly, the curves showed in Figure 6 were obtained through the numerical integration of the data shown in Figure. 4.
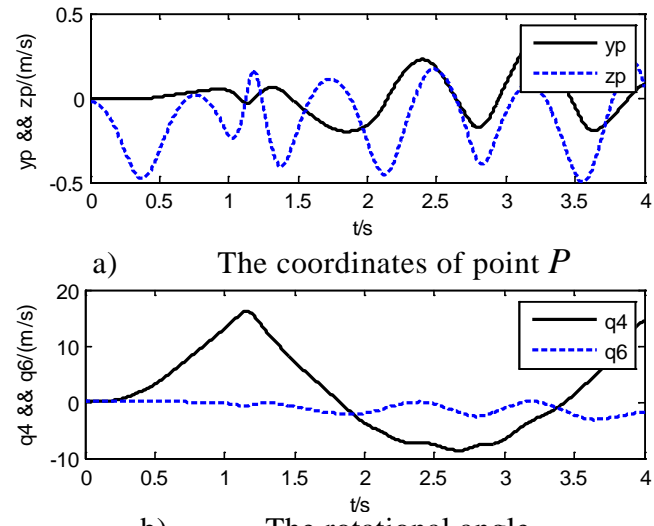

b) The rotational angle

FIGURE VI. THE POSITION COORDINATES AND THE ROTATIONAL ANGLE

Figure 7 shows the input torques of $B_{2}, B_{3}$ and $B_{5}$.

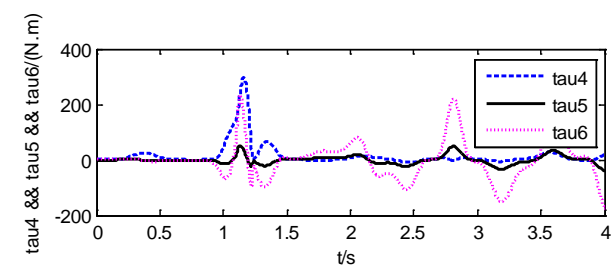

FIGURE VII. THE COMPARISON OF TWO KINDS OF KINETIC ENERGY

The results in Figure.1 Figure 7 show that the robot can run on the flexible wire, and the maximum torque on $B_{2}, B_{3}$ and $B_{5}$ is less than 350 N.m.

Furthermore, compare the difference between the energy increment of the system and the external forces' elementary work, and the results are show in Figure 8.
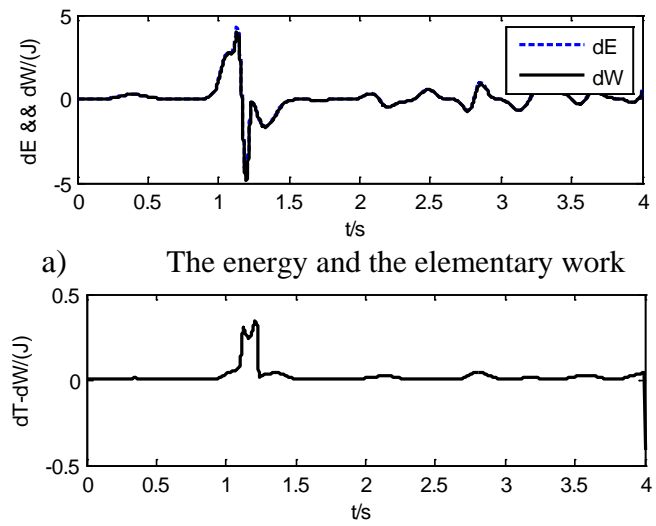

b) The difference of the energy increment and the elementary work

FIGURE VIII. THE ENERGY VS THE ELEMENTARY WORK 
As it is seen in Figure 8, while calculated by use of different variable, the energy increment of the system and the external forces' elementary work are coincident, and their difference is less than $10 \%$. The analysis shows the fact that the dynamical model (22) completely obey the law of conservation of energy, so to a certain extent, the derivations in this paper are reliable.

\section{CONCLUSIONS AND FUTURE WORK}

One of the work of this paper is that a novel wire-walking robot with swing-arms was designed. The other work is that a dynamical model for the system running on a flexible wire was explored in detail. The results show that the proposed wire-walking robot is an under-actuated system, which consist of seven independent velocities and three drivingtorque inputs. With the inverse dynamics simulation of a balanced running, the mechanism structure scheme of the robot system and the reliability of the dynamical model were validated.

By now in this paper, there are lack of physical experiments that can provide enough supports for the mentioned analysis, so the next work should focus on performing a balanced motion of the robot system.

\section{ACKNOWLEDGMENT}

Our work has received a great deal of financial support from National Natural Science Foundation of China (Grant No. 61365012 \&\&No.51305087\&\&No. 51765011) and Director Foundation of Guangxi Key Laboratory of Manufacturing Systems and Advanced Manufacturing Technology (Grant No. 14-045-15- 003Z).

\section{REFERENCES}

[1] Chunlin Z, Shiyi C, Jiaren S, et al. A kind of wire-moving robot. CN 1472044A, Apr 04, 2004.

[2] Song Jiewen, Jia Pengfei, Huang Wenxian, Li Gen, "A Simple Fuzzy Controller for Wire-Walking Robot,” China's scientific and technical papers online, Available: http://www.paper.edu.cn.

[3] Guanglei L, Lei G, Shimin W, et al. Dynamic modelling and simulation of wire- walking robot based on the control of balancing pole. IEEE. International Conference on Modelling Identification and Control (ICMIC). 2012 June 24-26; Wuhan, China. IEEE : pp.1329-6.

[4] Lei Guo, Xinhu Mo , Yuan Song and Ying Zhang. Balancing Control of A Kind of Wire-moving Robot. Proceeding of the 2015 IEEE International Conference on Information and Automation Lijiang, China, August 2015.

[5] Xinhu Mo, Lei Guo and Yuan Song. Sliding Mode Control of A Wiremoving Robot Based on Hurwitz Stability. Proceedings of the IEEE International Conference on Information and Automation Ningbo, China, August 2016.

[6] GUO Lei, MO Xinhu*, SONG Yuan. Dynamic Modeling and Adaptive Controller Design for a Wire-moving Robot. Proceedings of the 34th Chinese Control Conference July 28-30, 2015, Hangzhou, China

[7] Lei G, Shimin W, Guanglei L, et al. a rigid and flexible coupling wirewalking robot based on balance bar control. CN102424075A, Apr 04, 2012.

[8] L. Guo, G. Lu, Q. Liao, et al., Structure Design and Stablebalancing Control of a Kind of Wire-moving Robot, International Journal of Advanced Robotic Systems on Mobile Robots and Multi-Robot Systems, 11(78): 2014. 